La revue La revue pour l'histoire du CNRS

POUR L'HISTOIRE DU CNRS $\quad 6$ | 2002

Les années 60 : I'Espace, l'Océan, la Parole

\title{
La part du CNRS dans les débuts de la conquête de l'espace (1945-1965)
}

\section{Philippe Varnoteaux}

\section{(2) OpenEdition}

\section{Journals}

Édition électronique

URL : https://journals.openedition.org/histoire-cnrs/3601

DOI : 10.4000/histoire-cnrs.3601

ISSN : 1955-2408

Éditeur

CNRS Éditions

Édition imprimée

Date de publication : 5 mai 2002

ISBN : 978-2-271-05926-0

ISSN : $1298-9800$

\section{Référence électronique}

Philippe Varnoteaux, «La part du CNRS dans les débuts de la conquête de l'espace (1945-1965) », La revue pour l'histoire du CNRS [En ligne], 6 | 2002, mis en ligne le 05 juillet 2007, consulté le 20 mai 2021. URL : http://journals.openedition.org/histoire-cnrs/3601 ; DOI : https://doi.org/10.4000/histoire-cnrs. 3601

Ce document a été généré automatiquement le 20 mai 2021.

Comité pour l'histoire du CNRS 


\title{
La part du CNRS dans les débuts de la conquête de l'espace (1945-1965)
}

\author{
Philippe Varnoteaux
}

1 Si l'idée d'explorer l'espace est formulée dans de nombreux pays avant la Seconde

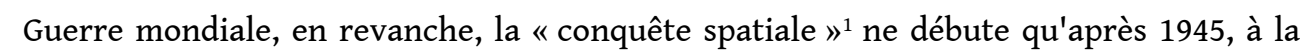
suite du pillage technologique de l'Allemagne nazie. Parmi le matériel récupéré figurait la célèbre fusée $\mathrm{V}^{2}$. Celle-ci est alors une arme de guerre révolutionnaire que tous les Alliés cherchent à acquérir, puis à perfectionner³. C'est la raison pour laquelle la recherche sur les fusées est immédiatement confisquée par les militaires. Parallèlement, la communauté scientifique internationale propose la tenue d'une «année géophysique internationale (AGI)», calquée sur le modèle des expéditions polaires, afin d'étudier la haute atmosphère ${ }^{4}$. C'est dans ce double contexte que la conquête de l'espace va prendre corps.

2 En France, de nombreux scientifiques souhaitent participer à cette AGI, mais comment aller étudier la haute atmosphère? Les ballons existent bien ${ }^{5}$, mais la fusée apparaît rapidement comme l'outil technologique idéal, car elle peut sonder " l'océan aérien " $^{6}$ à de plus hautes altitudes, certes moins longtemps qu'un ballon?

Intéressés, les militaires français accepteront de mettre à la disposition des scientifiques la première fusée-sonde opérationnelle : Véronique. Ainsi, l'exploration de l'atmosphère pourra commencer. Le CNRS est l'un des tout premiers organismes scientifiques qui se lancent dans l'aventure. Il jouera un rôle de premier plan, et, lorsque se définit la politique spatiale nationale (1961-1962) au sein du CNES, ses laboratoires et ses chercheurs fournissent le principal effort et contribuent à faire de la France, le 26 novembre 1965, la troisième puissance spatiale ${ }^{8}$.

La contribution du CNRS dans la recherche technique des fusées au lendemain de la Seconde Guerre mondiale

4 Trois mois après le débarquement de Normandie, le directeur du Laboratoire municipal de Paris, le professeur Henri Moureu ${ }^{9}$, découvre l'existence de la fusée V2 ${ }^{10}$. En 1945, au cours de missions scientifiques ${ }^{11}$, il rapporte d'Allemagne de la documentation et des éléments de fusée. Ses recherches passionnent au plus haut point les militaires qui lui 
apportent une aide logistique. Dans un rapport daté du 23 décembre 1946, il fait l'apologie de la fusée balistique et incite fortement le gouvernement à soutenir toutes les recherches qui pourraient être entreprises dans ce domaine. Il avance deux principales raisons: d'un point de vue militaire, la fusée révolutionnera immanquablement les guerres de l'avenir, surtout si on lui associe la bombe atomique; enfin, au niveau scientifique, la fusée "va permettre d'étudier la haute atmosphère et les phénomènes particulièrement complexes dont elle est le siège ${ }^{12}$ ». En effet, pour lui, il ne fait aucun doute qu'elle offrira une opportunité extraordinaire aux scientifiques qui cherchent à approfondir la connaissance des phénomènes atmosphériques, que ce soit pour la météorologie, la physique de l'atmosphère ou pour d'autres domaines, comme la géophysique.

5 Dès le 26 juillet 1945, H. Moureu propose la création d'un organisme d'État chargé de développer les recherches sur la fusée. Son projet aboutit le 24 août, sous le nom de Centre d'études des projectiles autopropulsés (CEPA). Des scientifiques proches de $\mathrm{H}$. Moureu, par exemple les professeurs Chaudron et Daudel de la faculté des sciences de Paris, saluent l'événement et le rejoignent immédiatement. Étant donné que la fusée relève à ce moment-là des militaires, le CEPA est placé directement sous la responsabilité de la direction des études et fabrication d'armement (DEFA) de l'armée de terre. Le professeur Moureu recrute des scientifiques de haut niveau. Certains viennent du CNRS, comme le professeur Lévy, directeur du Service central de microanalyse, qui est chargé de conduire des études sur les propergols et qui contribue à l'amélioration des connaissances sur la propulsion des fusées. Il est démontré que, dans le cas des futures fusées spatiales, la propulsion à liquides semble mieux adaptée que celle des poudres, car elle délivre une poussée plus longue et mieux contrôlée au cours du vol.

6 Malheureusement, les problèmes financiers et les difficultés technologiques ${ }^{13}$ conduisent à une crise en 1947 : la plupart des militaires ne veulent plus de la fusée balistique en tant qu'engin opérationnel, car trop complexe à mettre en oeuvre, et, surtout, ils ne souhaitent pas léser les autres systèmes d'armes plus traditionnels. C'est l'échec du CEPA et, provisoirement, de la fusée. Cependant, des groupes d'études existant déjà, et pour ne pas perdre totalement ces recherches, une veille technologique est maintenue; par exemple, au LRBA de Vernon ${ }^{14}$ s'ébauche une mini-V2 très rudimentaire : la fusée Véroniqu $e^{15}$.

Le développement de la collaboration entre les militaires et les scientifiques

7 À la fin de la Seconde Guerre mondiale, les militaires prennent acte de l'extraordinaire avance technologique de l'Allemagne ${ }^{16}$. Cependant, s'ils souhaitent rattraper le retard, et ne pas se laisser distancer par les Américains et les Soviétiques, ils doivent établir d'urgence une étroite " collaboration avec les hommes de science pour l'amélioration de la technique des armes modernes ${ }^{17}$ ». Est alors émis le voeu de travailler « en étroite liaison avec le $\mathrm{CNRS}^{18}$ ». Pourquoi le CNRS ? Créé en 1939, le CNRS voit son statut d'organisme fédératif de la recherche confirmé en novembre 1945, tout en étant chargé d'entreprendre de la prospective et d'accroître la coopération avec d'autres organismes, y compris militaires ${ }^{19}$. De ce fait, il devient le coeur et le symbole de la recherche scientifique en France.

8 Afin de faciliter la coopération avec les militaires, le directeur du CNRS, Frédéric JoliotCurie $^{20}$, propose la création du Comité de coordination scientifique de la Défense nationale (CCSDN), organisme qui doit rapprocher les différents acteurs ${ }^{21}$. La démarche 
ne doit pas surprendre, car si les scientifiques tiennent à collaborer avec les militaires, c'est aussi pour obtenir des crédits de recherches supplémentaires. N'oublions pas qu'au lendemain de la Libération, l'État est financièrement exsangue. De plus, les nécessités de la reconstruction d'une armée nationale font que les militaires disposent d'importants crédits. Dès lors, pourquoi ne pas unir les efforts? Les trois armées approuvent la proposition du directeur du CNRS. Les militaires admettent également qu'il faut favoriser d'urgence les liaisons entre scientifiques et militaires, et entre chercheurs et utilisateurs, car les leçons de la dernière guerre montrent que, désormais, les armes seront de plus en plus performantes et technologiques et nécessiteront davantage l'aide des scientifiques ${ }^{22}$.

Toutefois, la collaboration interarmées se met difficilement en place, car chacun estime que l'autre a suffisamment de crédits et de chercheurs. Le CCSDN n'arrive pas à établir un climat de confiance suffisant ${ }^{23}$. Pour éviter la paralysie, le CCSDN se transforme le 24 mai 1948 en un organisme plus autonome et avec davantage de compétences : le Comité d'action scientifique de la Défense nationale (CASDN $)^{24}$. Le principal objectif est de favoriser toutes les collaborations et d'aider les projets jugés les plus intéressants en fournissant les scientifiques spécialisés. Cependant, entre 1948 et 1953, le CASDN est lui aussi souvent neutralisé en raison des querelles et des jalousies entre les armées. Les scientifiques subissent. La situation ne s'améliore qu'à partir des années 1954-1955 sous l'action du général Bergeron, le président du CASDN (1948-1955) ${ }^{25}$.

10 De son côté, l'État finit par prendre conscience des enjeux : en 1954, est créé, auprès de la Présidence du Conseil, le Conseil supérieur de la recherche scientifique et du progrès technique (CSRSPT). Ce Conseil prend l'allure d'un véritable "gouvernement de la science» et il est chargé d'assurer les investissements, d'élaborer une politique scientifique nationale. Toutefois, si la collaboration entre les scientifiques et les militaires se développe progressivement, le «jeu» n'est pas systématiquement respecté par tout le monde, à commencer par l'armée de l'air. Cette dernière fait souvent sécession en s'appuyant davantage sur son propre vivier de scientifiques, regroupés notamment au sein de l'office national des études et de recherches aéronautiques (ONERA) ${ }^{26}$. Cette situation entraîne une déperdition inutile des efforts, car les trois armées s'enferment dans leurs bureaux d'étude respectifs.

11 À partir de 1958, le Comité d'action scientifique réussit enfin à s'imposer. Il parvient à réunir en son sein les meilleurs scientifiques de l'époque ${ }^{27}$. Ceux du CNRS tiennent une place privilégiée : «Les militaires du CASDN tenaient tout particulièrement à travailler avec des scientifiques provenant d'organismes prestigieux comme le CNRS qui représentait un peu la science française ", nous rappelait il y a quelques années Arlette Vassy ${ }^{28}$. Parmi les principales personnalités rejoignant le CASDN figure le professeur Joseph Pérès, mathématicien, spécialiste de la mécanique des fluides, membre de l'Académie des sciences et directeur adjoint du CNRS. En quelques années se forme ainsi un véritable brain trust qui apprend à travailler avec les militaires ${ }^{29}$.

De l'exploration de la haute atmosphère à la conquête de l'espace

12 Dans le même temps, les militaires prennent conscience qu'il devient indispensable de mieux comprendre les phénomènes atmosphériques, car les avions et les nouvelles armes autopropulsées évoluent de plus en plus haut dans l'atmosphère. Ainsi, de nouvelles études s'engagent dans l'aérodynamique, la résistance des matériaux, la mécanique des fluides ou encore l'étude des particules. En effet, le dernier conflit venait de révéler l'importance de l'ionosphère, une couche particulière de la haute 
atmosphère dans laquelle se propagent les ondes radios: "Juste après la guerre, souligne la physicienne A. Vassy, les militaires sont venus nous chercher. Ils avaient besoin de nous pour améliorer leur connaissance sur l'ionosphère. Or, la maîtrise des communications, vitale pour les armées, passait obligatoirement par l'étude de cette ionosphère ${ }^{30}$. Les militaires ont donc un besoin urgent de spécialistes en la matière. Par exemple, dès 1945, la Marine met en place son propre bureau de recherche sur l'ionosphère qu'elle confie au physicien Yves Rocard ${ }^{31}$.

13 En 1954, les circonstances permettent la convergence des études sur la haute atmosphère avec la fusée, jusqu'alors "boudée » par les scientifiques. De plus, à la même époque, les militaires ne savent pas comment légitimer financièrement la fusée Véronique, étant donné qu'elle n'intéresse ni l'État ni les états-majors. C'est le professeur Étienne Vassy ${ }^{32}$ qui débloque la situation, avec le soutien du général Bergeron : pourquoi ne pas utiliser la fusée Véronique pour mener des études dans la haute atmosphère ? L'idée séduit immédiatement. Les premiers tirs expérimentaux de février et d'octobre 1954 démontrent l'extraordinaire avantage de la fusée, capable d'embarquer des instruments de mesures à de hautes altitudes, en très peu de temps ${ }^{33}$. Peu à peu, la communauté scientifique française accorde davantage de crédit à la fusée, considérée jusqu'alors uniquement comme un outil militaire.

14 Si les esprits changent, c'est également grâce à l'avènement de l'AGI. Des scientifiques appartenant à différents pays souhaitent organiser une vaste exploration de la haute atmosphère, au moment où l'activité solaire sera à son maximum en 1957-1958. Il s'agit de mieux cerner les interactions des rayonnements du soleil avec l'atmosphère terrestre. Pour faciliter la participation française à cette manifestation, le gouvernement met en place une commission « année géophysique » en janvier 1956. La responsabilité en incombe à André Danjon, directeur de l'Observatoire de Paris. Le CNRS y tient une part prépondérante, car son directeur, Gaston Dupouy, le seconde.

En juillet 1957 s'ouvre l'AGI. En octobre et novembre, l'Union soviétique crée la double surprise en plaçant sur orbite Spoutnik-1 et 2, les premiers satellites artificiels de l'histoire, avant même les Américains. L'exploration de la haute atmosphère prend soudainement une nouvelle dimension, d'autant plus importante que le second Spoutnik est occupé par un animal, la chienne Laïka. Le message soviétique est clair: la prochaine étape est l'envoi d'un homme dans l'espace. De ce fait, les manifestations de l'AGI sont de plus en plus occultées par les aspects technologiques et militaires des satellites et des fusées. Métamorphosée en conquête de la proche banlieue terrestre, l'exploration de la haute atmosphère bascule en un furieux affrontement idéologique entre les deux grandes puissances ${ }^{34}$. Comment la France se positionne-t-elle?

Le CNRS et les premiers programmes spatiaux

En raison du retard pris dans le développement d'une version plus performante de Véronique (Véronique-AGI), la France n'entame ses premières expériences spatiales qu'en mars 1959. Si la charge technique des fusées incombe au Centre national d'études des télécommunications (CNET), la responsabilité des expériences scientifiques (embarquées dans les fusées-sondes) revient au CNRS. En effet, il n'existe pas encore une réelle agence spatiale, même s'il vient de se mettre en place, en janvier 1959, un Comité des recherches spatiales. Comme le soulignera plus tard Jean Coulomb ${ }^{35}$, il fallait rapidement entreprendre quelque chose en faisant «appel au milieu universitaire où l'on trouve, où l'on devrait trouver, sinon des spécialistes de chaque question, du moins des hommes qui en soient curieux » et, surtout, recruter de jeunes 
chercheurs, car ainsi « l'avenir serait assuré $»^{36}$. Dans cette optique, J. Coulomb instaure un service d'aéronomie au sein du CNRS et en offre la direction au prestigieux prix Nobel de physique Alfred Kastler. Quant à la réalisation des expériences, elle est confiée à son jeune directeur adjoint, le professeur Jacques Blamont ${ }^{37}$ qui, en mars 1959, fait la « une » des quotidiens avec le lancement de deux fusées Véronique-AGI : il réalise des nuages de sodium en haute altitude, approfondissant ainsi les connaissances concernant les variations de température et de pression, mais aussi le déplacement des vents $^{38}$.

Le succès est tel qu'il permet d'une part aux scientifiques français de rejoindre les deux superpuissances dans la course à l'espace et, d'autre part, de convaincre le nouveau pouvoir - le général de Gaulle a repris la tête du gouvernement depuis 1958 - d'offrir aux scientifiques plus de moyens, de cohésion, mais aussi de rayonnement. Pour cela, de nouvelles structures sont constituées. Tout d'abord, au niveau de la recherche scientifique est créé le Comité consultatif de la recherche scientifique et technique (CCRST) qui propose, à travers les comités interministériels (CIRST), des actions et des aides financières pour aider le développement de la recherche ${ }^{39}$. Rapidement, les réunions du CCRST ne suffisent plus. C'est la raison pour laquelle apparait la Délégation générale à la recherche scientifique et technique (DGRST), chargée d'établir un suivi des affaires et d'en tenir informé le gouvernement. Ainsi, depuis le retour au pouvoir du général de Gaulle, la recherche est devenue une prioritée ${ }^{40}$. C'est d'ailleurs au cours d'une réunion du CCRST qu'avait été décidée la création d'un Comité des recherches spatiales, confié au professeur Pierre Auger. Ce dernier devait rassembler les forces vives du pays engagées dans l'aventure spatiale et les combiner avec les intérêts de l'Etat. Le CNRS ne pouvait sérieusement tenir seul ce rôle; ce n'était pas sa vocation.

Parallèlement, les campagnes de tirs de fusées-sondes s'accélèrent (7 Véronique en 1960; 6 Véronique, 7 Centaure et 2 Bélier en 1961). De nouveaux acteurs civils et militaires rejoignent l'aventure spatiale: la Météorologie nationale, le Centre d'études et de recherches en médecine aéronautique (CERMA), ou encore le CEA. Le Comité des recherches spatiales apparaît vite insuffisant, parfois dépassé. C'est la raison pour laquelle est enfin créé le CNES, Centre national d'études spatiales, le 19 décembre $1961^{41}$. Le nouvel organisme canalise, puis centralise progressivement l'ensemble des recherches scientifiques et techniques liées au spatial.

$\mathrm{Au}$ moment où est défini le premier plan programme scientifique du CNES (1962), prévu pour les années 1962-1965, le CNRS déploie une véritable «offensive de charme » : il incite en effet ses laboratoires à s'investir plus avant dans le spatial. Le service d'aéronomie occupe toujours la première place dans ces activités. L'équipe du professeur J. Blamont poursuit avec brio les expériences au sodium. De nombreuses fusées-sondes sont lancées afin de poursuivre l'étude des couches atmosphériques situées entre 90 et $250 \mathrm{~km}$ d'altitude ${ }^{42}$. Outre le service d'aéronomie, on trouve le Laboratoire de physique cosmique (LPC) et le Laboratoire d'aérothermique (LA). Le LPC présente un ambitieux programme concernant l'étude du rayonnement cosmique. Pour le réaliser, il travaille en étroite collaboration avec les observatoires de Paris et du pic $\mathrm{du}$ Midi. Le projet inclut également une coopération européenne avec une équipe néerlandaise ${ }^{43}$. Les expériences sont réalisées avec des ballons et des fusées-sondes. Ainsi, en avril et en novembre 1964, plusieurs fusées Véronique sont lancées avec succès pour étudier les rayonnements cosmiques et solaires. De son côté, le LA se penche, d'une part, sur le problème de la frontière entre l'espace et la haute atmosphère 
terrestre et, d'autre part, sur l'approfondissement des connaissances sur la basse densité de l'atmosphère. Ce dernier domaine est particulièrement important, car il permet d'en savoir plus sur les problèmes de rentrée orbitale d'engins. Cela intéresse au plus haut point les militaires qui, dans le même temps, développent le missile balistique pour la force de frappe nucléaire française à travers la Société pour l'étude et la réalisation d'engins balistiques (SEREB). La collaboration avec les armées se place désormais au plus haut niveau et, en échange, les scientifiques bénéficient des retombées technologiques militaires en obtenant un de leur missile : Diamant. Celui-ci devient le premier lance-satellite français, qui place sur orbite le premier satellite national A-1, le 26 novembre 1965.

Au cours des années 1960, le CNRS a donc joué un rôle de premier plan dans le développement des programmes spatiaux. La preuve en est que, dès le 31 octobre 1962, le directeur général du CNRS, J. Coulomb, est nommé président du CNES. D'autres responsables du CNRS occuperont cette place, comme Hubert Curien (de 1976 à 1984) ou encore René Pellat (de 1992 à 1995).

Le CNRS et l'espace, 40 ans plus tard

21 Si le nombre des organismes s'investissant dans la conquête de l'espace a considérablement augmenté depuis cette époque, le CNRS a continué à développer de nouvelles unités de recherche comme l'Institut d'astrophysique spatiale (IAS), le Laboratoire de météorologie dynamique (LMD), le Laboratoire de physiologie neurosensorielle (LPN), le Laboratoire d'astronomie spatiale (LAS), ou encore le Centre de recherche en physique de l'environnement terrestre et planétaire (CRPE). Par exemple, le CRPE est engagé dans des programmes spatiaux ambitieux comme la sonde européenne Ulysse, qui, depuis octobre 1990, étudie la topologie des vents solaires. Le LAS embarque régulièrement des instruments dans des satellites, comme la sonde russe Véga en direction de la comète de Halley (1984), ou dans les navettes spatiales américaines, comme des télescopes pour l'observation du rayonnement ultraviolet. Quant au LPN, il est étroitement associé aux vols habités français, en coopération avec la Russie et les États-Unis, notamment sur l'étude des fonctions sensori-motrices en apesanteur, l'irradiation des tissus vivants ou encore sur le comportement visiomoteur des spationautes français.

Ainsi, les centres de recherche du CNRS se sont fait une spécialité dans les domaines de la biologie spatiale et de l'étude du système solaire. L'espace est devenu un champ de recherche scientifique à part entière.

\section{NOTES}

1.L'expression « conquête de l'espace » se généralisera après le lancement de Spoutnik-1 par l'URSS le 4 octobre 1957 ; avant cette date, on parle plutôt « d'exploration ». Voir à ce sujet notre article « Les réactions internationales au lancement de Spoutnik-1, le premier satellite artificiel de l'histoire », Les Cahiers de Mars, 1er trimestre 2000, p. 133-139. 
2.Si le pillage technologique de l'Allemagne par les États-Unis est désormais bien connu (voir L. Hunt, L'Affaire Paperclip, Stock, Paris, 1995), le pillage par les Français l'est moins ; soulignons qu'il a été tout aussi important (voir notre article « Les missions scientifiques ou comment la France a découvert, puis récupéré les armes secrètes nazies ", Histoire de guerre, décembre 2000, p. 74-78.

3.Conçue par le célèbre ingénieur allemand von Braun, la fusée V2, c'est-à-dire « arme de représailles $n^{\circ} 2 »$, est considérée comme le premier engin balistique de l'histoire. Parmi les plus récentes publications, voir S. Foiret, «V2 Aufbruch zur Raumfahrt », Icare, $\mathrm{n}^{\circ} 148$, 1er trimestre 1994, p. 45-47 ; O. Huwart, « Les héritages français de la V2 », Le Fana de l'aviation, $\mathrm{n}^{\circ} 365$, avril 2000, p. 44-51; ce dernier achève actuellement une thèse de doctorat portant sur les VI et V2.

4.L'idée de l'AGI est née lors de la conférence de Bruxelles, en 1950, sur la proposition du scientifique américain Berkner. Un comité de l'ionosphère est créé et chargé d'organiser et de coordonner tous les projets scientifiques. Les manifestations sont prévues pour les années 1957-1958, au moment où le soleil aura le plus d'incidences dans l'atmosphère terrestre. C'est dans ce contexte que les Américains et les Soviétiques feront des surenchères de plus en plus spectaculaires et finiront par annoncer le lancement de plusieurs centaines de fusées-sondes et de quelques satellites artificiels. Sur l'AGI, voir W. Buedeler, «L'année géophysique internationale », in L'Unesco et son programme, XV, Paris, Unesco, 1957.

5.En France, le professeur Picard utilise les ballons pour explorer la haute atmosphère : le 2 septembre 1947, il atteint l'altitude de 30000 mètres. Son exploit sera suivi par l'astronome A. Dollfus, qui effectuera même de observations de la planète Mars depuis une nacelle de ballon. À ce sujet, voir A. Dollfus, 50 ans d'astronomie, Paris, EDP Sciences, 1998.

6.Termes employés par P. Rousseau, in Satellites artificiels, Paris, Hachette 1957, p. 16. 7.Cela explique qu'encore aujourd'hui, le CNES utilise la technique des ballons dans la conquête de l'espace comme le télescope submillimétrique PRONAOS, qui a volé à plusieurs reprises $(1994,1996,1999)$ avec des instruments du CNRS (spectromètre photométrique multibande).

8.Ce jour-là, la France place avec succès sur orbite le premier satellite national A-1. 9.Docteur ès sciences physiques en 1930, il devient en 1937 sous-directeur du Laboratoire de chimie et physique nucléaire au Collège de France, puis, à partir de 1941, directeur du Laboratoire municipal de Paris.

10.Rappelons que les premiers V2 ne tombent pas sur Londres mais près de Paris. À ce sujet voir les travaux de Jacques Villain, notamment La France a-t-elle hérité de Peenemünde ?, Paris, publication SEP, octobre 1992.

11.Termes consacrés à l'époque pour désigner les groupes chargés de piller l'Allemagne vaincue.

12.In Rapport n 9698/CEPA, SHAA, Service historique de l'armée de l'Air, fonds Hautefeuille, Z.32.605, dossier 3/3.

13.De nombreuses difficultés apparaissent en effet au niveau du pilotage des engins, du choix des ergols, de l'effet Pogo (tremblements au moment du décollage), du contrôle de vol et de la trajectoire, très problématique sans la maîtrise de la télémesure, etc. 14.Le Laboratoire de recherches balistiques et aérodynamiques, situé à Vernon, dépendait de la DEFA de l'armée de terre. L'armée de l'air maintenait également des études de fusée avec notamment des engins SE-4100 (fusée ailée ressemblant au V2), SE-4400 (engin-fusée supersonique) ; sur ces engins, voir les récents travaux de Philippe 
Jung lors des derniers congrès d'astronautique de l'IAF « The true beginnings of French Astronautics (1938-1959) ", communication lors du congrès international d'Astronautique (IAF) 2000, «The SE-4400/4401 family : an hypersonic ramjet in the fifties ", 38e congrès IAF, Bangalore, Inde, 1988, « SNCASE Cannes rochets of the 50's », 47 congrès IAF, Beijing, Chine, 1996.

15.Alors que le projet Super V2 du LRBA de Vernon proposait un engin ayant une poussée de 40 tonnes, celui de Véronique se limitait à seulement 4 tonnes.

16.Pendant la guerre, les scientifiques allemands ont étroitement collaboré avec les militaires, permettant à ces derniers de réaliser de nombreuses armes révolutionnaires (bombe planante, avion sans pilote, fusée...). En retour, les scientifiques ont bénéficié d'importants crédits militaires, favorisant ainsi des domaines de recherche variés (ionosphère, radiocommunication...).

17.Lettre du ministre de l'Air, datée du 4 jan.vier 1945, in SHAH, E. 4398.

18.Ibid.

19.Voir à ce sujet Girolamo Ramunni, «La mise en place d'une politique scientifique », Actes du colloque De Gaulle en son siècle, tome 3, Moderniser la France, Paris, Plon, 1992, p. 654-712. Sur les débuts du CNRS, voir Jean-François Picard et Élisabeth Pradoura, «La longue marche vers le CNRS ", Cahiers pour l'histoire du CNRS, n 1, CNRS Éditions, 1989, p. 7-40.

20.Spécialiste en physique nucléaire, F Joliot-Curie (1900-1958) devient en 1946 le premier responsable du Commissariat à l'énergie atomique (CEA) où il élabore la première pile atomique française (1948).

21.Les recherches de François Jacq (voir sa thèse de doctorat Pratiques scientifiques, formes d'organisation et conceptions politiques de la science dans la France d'après-guerre. La "politique de la science » comme énoncé collectif, Paris, École des Mines, 1996) ont récemment démontré que F. Joliot-Curie est bien l'instigateur du CCSDN.

22.Dès 1948, dans son ouvrage Les Armes de demain, (Paris, Berger-Levrault), le chroniqueur scientifique Albert Ducrocq souligne que « les guerres de demain se feront sous l'angle de la technique moderne » (p. 8). Deux ans plus tard, l'ingénieur civil des Mines, H.-J. Proumen, tient les mêmes propos dans son livre, Armes nouvelles dans une guerre future, Paris, O. de Publicité, 1950.

23.On trouve des traces de méfiance notamment dans un rapport tardif intitulé "Pourquoi une liaison intime Recherche-Armée de l'air », sans date précise (1947 ou 1948), in Service historique de l'armée de l'air, E. 4397.

24.Le CCSDN fonctionnait selon le bon vouloir des états-majors des trois armées, sans réelle coordination. Le projet CASDN a été présenté par le général Paul Dassault, président du CCSDN, qui regrettait la paralysie de son organisme.

25.Voir à ce sujet notre article, « Au coeur de la modernisation des armées, il y a 50 ans le CASDN », Armées d'aujourd'hui, n 231, juin 1998, p. 72-74.

26.L'ONERA a été créé le 3 mai 1946 dans l'optique de reconstituer rapidement l'aéronautique française.

27.C'est ce que nous avons démontré dans le chapitre 4 de notre thèse intitulée «Les origines et les enjeux de la conquête de l'espace en France (1944-1962) », p. 187-231. En effet, les militaires du CASDN réussissent à établir une profonde et durable interpénétration des projets scientifico-militaro-industriels qui paraissent utopiques à l'époque et qui finissent par devenir une réalité. Parmi les scientifiques de renom associés au CASDN, on trouvait le physicien Louis de Broglie, le mathématicien et 
physicien Yves Rocard, l'astronome André Danjon, ou encore le physicien Louis Leprince-Ringuet.

28.Déclaration d'A. Vassy à l'auteur le 22 novembre 1996. Docteur ès sciences physiques en 1941, elle devient maître de recherche au CNRS en 1954. Pendant l'AGI, elle est responsable de la Commission de l'ozone du Comité national français.

29.Voir notamment R. Hautefeuille et A. Teyssier, « Recherche scientifique et politique militaire en France (1945-1958) », Revue historique des armées, n 175, juin 1989, p.

111-122.

30.Déclaration d'A. Vassy à l'auteur (1996).

31.Voir à ce sujet Dominique Pestre, «Études de l'ionosphère et prévisions pour les radiocommunications » in L'Essor de la politique spatiale française dans le contexte international (1958-1964), sous la direction de Maurice Vaisse, Amsterdam, EAC, 1997, p. 1-30.

32.Titulaire d'un doctorat ès sciences, professeur à la faculté des sciences de Paris, E. Vassy rejoint H. Moureu au CEPA, puis le CASDN en 1950.

33.Les Américains et les Soviétiques pratiquaient ce genre d'expérimentation depuis la fin des années 1940, mais les scientifiques français demeuraient sceptiques et les estimaient anecdotiques, comme le confirme A. Vassy : « Lorsque mon mari [le professeur E. Vassy] a proposé d'utiliser Véronique pour sonder la haute atmosphère, beaucoup de ses confrères ne l'ont pas pris au sérieux » (entretien avec l'auteur, 1996). 34.H. Pierre, «Washington : on s'inquiète plus de l'effet de propagande obtenu par Moscou que des répercussions militaires de l'expérience », Le Monde, 8 octobre 1957, p. 3.

35.Professeur de physique du globe à la Sorbonne (1941), J. Coulomb devient directeur général du CNRS en 1957, puis président du CNES de 1962 à 1967.

36.J. Coulomb, Bulletin d'informations du CNES, n 6, décembre 1962, p. 1.

37.Docteur ès sciences physiques, J. Blamont devient le responsable du Laboratoire d'aéronomie du Service d'aéronomie et de physique cosmique du CNRS (1958), avant de devenir le premier directeur scientifique du CNES (1961).

38.Le 10 mars 1959, il fait une réelle découverte : «Par chance, j'ai fait une découverte avec les premiers tirs de Véronique. J'ai mis en évidence la turbopause, qui est la limite entre la basse atmosphère à $100 \mathrm{~km}$ d'altitude et la haute atmosphère. On l'appelle turbopause, parce que l'atmosphère est mélangée au-dessous des $100 \mathrm{~km}$, tandis qu'audelà, elle n'est plus mélangée, c'est-à-dire qu'il n'existe plus de mouvements verticaux; le milieu devient hétérogène, stratifié en fonction du poids moléculaire » (entretien avec l'auteur, Paris, 25 mai 1996).

39.Pierre Lelong, "Le général de Gaulle et la recherche en France ", in Actes du colloque De Gaulle en son siècle, tome 3, Moderniser la France, Paris, Plon, 1992, p. 643-653.

40.Voir G. Ramunni, «La recherche : une priorité de la Ve République », in L'Essor de la politique spatiale française dans le contexte international (1958-1964), Amsterdam, EAC, 1997, p. 47-65.

41.Sur la mise en place du CNES, voir C. Carlier et M. Gilli, Les Trente Premières Années du CNES, Paris, Documentation française, 1994.

42.Par exemple, en mai et juin 1962, plusieurs fusées-sondes Centaure (construites par la société Sud-Aviation) sont lancées depuis le centre d'Hammaguir, en Algérie, afin de réaliser notamment des mesures de l'échelle spatiale des vents.

43.Rapport sur les activités spatiales pour 1962, Centre des archives contemporaines de Fontainebleau, CSRSPT, 92/0547, carton 5, liasse 3. 
INDEX

Mots-clés : espace

\section{AUTEUR}

\section{PHILIPPE VARNOTEAUX}

Philippe Varnoteaux est professeur d'histoire et de géographie. 